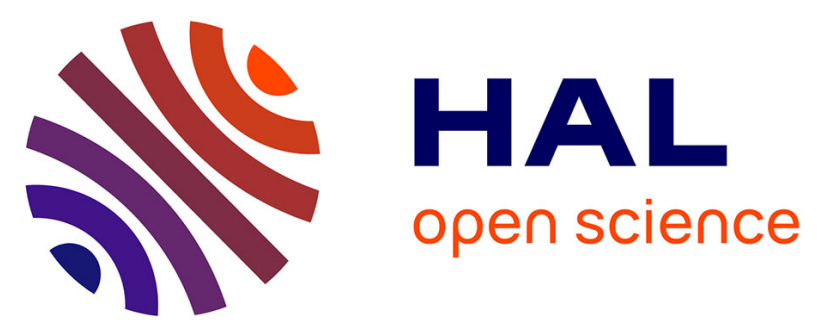

\title{
A general density-based index to analyze charge transfer phenomena: from models to butterfly molecules
}

Léon Huet, Anna Perfetto, Francesco Muniz-Miranda, Marco Campetella, Carlo Adamo, Ilaria Ciofini

\section{- To cite this version:}

Léon Huet, Anna Perfetto, Francesco Muniz-Miranda, Marco Campetella, Carlo Adamo, et al.. A general density-based index to analyze charge transfer phenomena: from models to butterfly molecules. Journal of Chemical Theory and Computation, 2020, 10.1021/acs.jctc.0c00296 . hal-02878834

\section{HAL Id: hal-02878834 \\ https://hal.science/hal-02878834}

Submitted on 23 Jun 2020

HAL is a multi-disciplinary open access archive for the deposit and dissemination of scientific research documents, whether they are published or not. The documents may come from teaching and research institutions in France or abroad, or from public or private research centers.
L'archive ouverte pluridisciplinaire HAL, est destinée au dépôt et à la diffusion de documents scientifiques de niveau recherche, publiés ou non, émanant des établissements d'enseignement et de recherche français ou étrangers, des laboratoires publics ou privés. 


\title{
A general density-based index to analyze charge
}

\section{transfer phenomena: from models to butterfly}

\section{molecules}

Léon Huet ${ }^{1+}$, Anna Perfetto ${ }^{1+}$, Francesco Muniz-Miranda ${ }^{1} *$, Marco Campetella ${ }^{l}$, Carlo Adamo $^{1,2}$, Ilaria Ciofini ${ }^{1} *$

Chimie ParisTech, PSL University, CNRS, Institute of Chemistry for Life and Health Sciences (i-CLeHS), Theoretical Chemistry and Modelling group (CTM), 75005 Paris, France and Institut Universitaire de France, 103 Boulevard Saint Michel, F-75005 Paris, France.

\begin{abstract}
We present a new formula and implementation for a descriptor enabling to quantify the electron-hole distance associated to a charge transfer of an optical transition, on the basis of the knowledge of the densities of the electronic ground and excited states. This index is able to define a charge-transfer length even for systems that would be otherwise difficult to treat, like symmetric molecules, while maintaining a very low computational cost and the possibility to be coupled to any method providing ground and excited state electron densities. After a benchmark of its performance on a series of push-pull molecules, the index has been applied to a set of large symmetric luminophores, the so-called "butterfly molecules", showing promising applications in optoelectronics, to highlight its potential use in the design of new compounds.
\end{abstract}


${ }^{\dagger}$ These two authors contributed equally.1) Chimie ParisTech; 2) IUF 


\section{Introduction}

Photoinduced charge-transfer (CT) and charge-separation are nowadays phenomena of paramount importance in many and diverse domains of applications ranging from energy production through light-harvesting, ${ }^{1}$ efficient chemoreceptive sensors, ${ }^{2}$ to organic fieldeffect transistors (OFETs). ${ }^{3}$ However, a complete experimental characterization of the electronic absorption processes could be complex, since the nature of the observed excitations is not always plainly evident. Time-resolved ${ }^{4}$ as well as two-colors UV-Vis experiments ${ }^{5}$ can yield additional information on the interplay between the various excited states and on their lifetimes, yet they require complex instrumentation and still cannot provide an orbital picture of excitation and de-excitation phenomena.

In this field, theoretical studies of photochemical and photophysical processes can give valuable and complementary insights on the inner processes of photon molecular absorption and emission. To achieve a deeper understanding of photophysical processes, an accurate characterization of the energetics and of the chemical nature of the electronic excited states is needed. Indeed, great efforts have been spent to develop quantum chemical approaches to this end. ${ }^{6,7}$ At the same time, this task could be made easier by the development of simple, and possibly computationally inexpensive, descriptors quantifying in a condensed way information about the nature of the electronic transitions involved.

The characterization of excited states often and routinely relies on the visualization and analysis of the (canonical) orbitals involved in the transitions. ${ }^{8-10}$ However, this approach may be not straightforwardly employed especially when many configurations are contributing to the excited states or the character of the orbitals involved in the transition is difficult to interpret and define. Furthermore, orbital analysis may be difficult to be directly connected to simple chemical parameters and concepts such as, for instance, charge transfer extent.

For these reasons, in the last decade several approaches have been derived to analyze the character of the excited states, and specifically to quantify the degree of locality of an excitation, meaning by this quantifying the hole-electron distance produced upon irradiation. Among others, one of the most applied is the so-called $\left(D_{C T}\right)$ index, which is a simple and intuitive measure of the CT distance. ${ }^{11}$ This index achieved great popularity and it has been adopted to investigate a wide range of very different molecular systems. Just to name a few, it has been used to characterize the optical response of BODIPY dyes, ${ }^{12}$ ruthenium and copper complexes, ${ }^{13-16}$ fluoride sensors, ${ }^{17}$ colorants for dye-sensitized solar cells, ${ }^{18-19}$ materials with enhanced delayed fluorescence, ${ }^{20}$ and the formation of radical pairs for biological 
applications. ${ }^{21}$ The wide application of the $D_{C T}$ index is due to its simplicity, effectiveness, and straightforward interpretation that allows it to be easily implemented into popular quantum chemical codes, such as Gaussian ${ }^{22}$ or MultiWFN. ${ }^{23}$

The $D_{C T}$ index is evaluated from the knowledge of the density difference between the two electronic states of interest. This descriptor is based on the analysis of the total electronic density in a real-space representation, ${ }^{11}$ computed for the ground and excited states and it allows to monitor the nature of the excited states. The $D_{C T}$ is indeed calculated as the module of the distance between the barycenters $\left(R^{+}\right.$and $\left.R^{-}\right)$of the charge density distribution corresponding to the hole and particle:

$$
D_{C T}=\left|\overrightarrow{R_{+}}-\overrightarrow{R_{-}}\right|
$$

Positive $\left(R_{+}\right)$and negative $\left(R_{-}\right)$barycenters are obtained by integration of the associated electron densities, $\rho^{+}$and $\rho^{-}$. These latter are derived from the difference in total density of the two states $S_{i}, S_{j}$ involved in the electronic transition - where $i=0, j>i$, with both $i$ and $j$ $\in \mathbb{N}$.

A drawback of the $D_{C T}$, already pointed out in the original paper, ${ }^{11}$ is that for symmetric system the barycenters of the hole and of the electron density distributions may coincide due to symmetry constraints, and thus the $D_{C T}$ will vanish even if a net charge-transfer occurs. As demonstrated by a recent paper, ${ }^{24}$ in such cases, the index may still be evaluated by identifying the corresponding symmetric irreducible subunits and limiting the density calculations on these latter.

Conversely, a great advantage of the $D_{C T}$ is that, since it relies only on the computed electronic densities for ground and excited state, it can be computed at any level of theory, such as density-functional and wave-function based methods, delivering the same qualitative description and quantitatively analysis of excited state evolution. ${ }^{25}$

Since then, many other descriptors have been developed in literature. For instance, Etienne et $a l .,{ }^{26-28}$ following the original approach introduced by Head-Gordon et al., ${ }^{8}$ have derived several descriptors to study the excited state topology by means of centroids of charges obtained from attachment/detachment density matrices. Another alternative descriptor was proposed by Guido et al., ${ }^{29} \Delta \mathrm{r}$, which relies on the calculation of the charge centroids of natural transition orbitals pairs. Peach and collaborators previously proposed an index that is as a diagnostic tool for failures of time-dependent density functional theory (TD-DFT), allowing to establish the reliability of the computed electronic transitions. ${ }^{30}$ Because the effects due to excitons formation may be crucial for a correct interpretation of the electronic 
excitations in solid-state physics, as well as for large molecular systems, ${ }^{31-33}$ Plasser et al. ${ }^{10,34-}$ ${ }^{36}$ have provided a general theory and comprehensive formalism for the correct evaluation of excitons properties. Their exciton analysis was initially based on population analysis, ${ }^{37}$ and later generalized. ${ }^{10,36,38}$ Based on the definition of the exciton wave function, and its corresponding electron-hole correlation function, Sharifzadeh and collaborators made contributions to quantify the extent and directionality of the solid-state exciton, and the associated degree of $\mathrm{CT}$ character. ${ }^{39}$ If on the one hand large real-space supercells are necessary for good convergence of the exciton wave functions, on the other side this analysis has the advantage to be general and applicable to quantify $\mathrm{CT}$ in complex molecular systems independently of their symmetry.

In order to further expand the domain of applicability of $D_{C T}$, which still remains the most used descriptor to estimate the CT length, the present paper is proposing a new formulation for the $D_{C T}$ index enabling the treatment of all systems independently of their symmetry. To this end, instead of defining the charge transfer distance as the distance between two average points of the hole and electron distribution (i.e. the two barycenters of charge distributions as in the $D_{C T}$ ), a novel density-based descriptor, the so called "Average Distance of Charge Transfer" $\left({ }^{A} D_{C T}\right)$ index, was designed as a weighted average of the distances between hole and electron distributions. As for the original $D_{C T}$, the ${ }^{A} D_{C T}$ is general and can be used in combination with any quantum-chemical method yielding electronic densities for the ground and excited states. The main advantage of ${ }^{A} D_{C T}$ is its general applicability to both symmetric and non-symmetric systems, without requiring an ad hoc definition of fragments. ${ }^{24}$ Anyway, contrary to the $D_{C T}$, being an average of distances, the index has no vectorial character (i.e. it is a scalar quantity).

This paper has been organized as follows. After the description of the new index and an explanation of technical points, the results obtained are reported in the Results and Discussion section first for some model compounds, namely a family of push-pull system that have been spectroscopically analyzed, and then for a set of large molecules, particularly interesting for their practical application in many different technologies like organic lightemitting diodes (OLEDs).

\section{Theoretical framework}

The original formulation of the $D_{C T}$ index $^{11}$ is extremely simple since it requires only the calculation of the electronic density of the ground $\left(\rho^{G S}\right)$ and excited state $\left(\rho^{E S}\right)$ of interest. The density change associated to the electronic transition $\Delta \rho(\boldsymbol{r})$ is given by: 


$$
\Delta \rho(\boldsymbol{r})=\rho^{E S}-\rho^{G S}
$$

From $\Delta \rho(\boldsymbol{r})$, one can define two functions $\rho^{+}$and $\rho^{-}$, which collect the points in the space where an increment or a depletion of electron density occurs upon excitation:

$$
\begin{gathered}
\rho^{+}(\boldsymbol{r})= \begin{cases}\Delta \rho(\boldsymbol{r}) & \text { if } \Delta \rho(\boldsymbol{r})>0 \\
0 & \text { if } \Delta \rho(\boldsymbol{r}) \leq 0\end{cases} \\
\rho^{-}(\boldsymbol{r})=\left\{\begin{array}{ll}
\Delta \rho(\boldsymbol{r}) & \text { if } \Delta \rho(\boldsymbol{r})<0 \\
0 & \text { if } \Delta \rho(\boldsymbol{r}) \geq 0
\end{array} .\right.
\end{gathered}
$$

From these two charge distributions, $D_{C T}$ can be expressed as the spatial distance between the two barycenters of the positive and negative density distributions as in Eq 1:

$$
\begin{gathered}
\boldsymbol{R}^{+}=\frac{\int \rho^{+}(\boldsymbol{r}) \boldsymbol{r} d \boldsymbol{r}}{\int \rho^{+}(\boldsymbol{r}) d \boldsymbol{r}}=\left(x_{+}, y_{+}, z_{+}\right) \\
\boldsymbol{R}^{-}=\frac{\int \rho^{-}(\boldsymbol{r}) \boldsymbol{r} d \boldsymbol{r}}{\int \rho^{-}(\boldsymbol{r}) d \boldsymbol{r}}=\left(x_{-}, y_{-}, z_{-}\right) . \\
\boldsymbol{D}_{C T}=\boldsymbol{R}^{+}-\boldsymbol{R}^{-} .
\end{gathered}
$$

$D_{C T}$ itself has, therefore, a vectorial character, being the difference between the positions of the two barycenters. This descriptor, measuring the effective distance of electron and the hole upon excitation, proved very useful to characterize CT in molecular systems, ${ }^{11-21,24-25,40-44}$ and, at the same time, effortless to calculate utilizing either density or wavefunction-based approaches.

This index can be also evaluated using some partitioning scheme of the density yielding atomic charges $\left(Q_{i}, i\right.$ being the $i^{\text {th }}$ atom $),{ }^{45-46}$ in which case equations (2)-(6) become:

$$
\begin{gathered}
\Delta Q_{i}=Q_{i}^{E S}-Q_{i}^{G S} \\
Q_{i}^{+}= \begin{cases}=\Delta Q_{i} & \text { if } \Delta Q_{i}>0 \\
=0 & \text { if } \Delta Q_{i} \leq 0\end{cases} \\
Q_{i}^{-}= \begin{cases}=\Delta Q_{i} & \text { if } \Delta Q_{i}<0 \\
=0 & \text { if } \Delta Q_{i} \geq 0\end{cases} \\
\boldsymbol{R}^{+}=\frac{\sum_{i} Q_{i}^{+} r}{\sum_{i} Q_{i}} \\
\boldsymbol{R}^{-}=\frac{\sum_{i} Q_{i}^{-} r}{\sum_{i} Q_{i}} .
\end{gathered}
$$


As mentioned, this index vanishes for symmetric molecules by construction. To overcome this limitation a way out is considering only the subset of atoms that constitutes the smallest asymmetric fragment. The ground (GS) and excited state (ES) densities can be expressed in terms of atomic basis functions $\left(\chi_{\alpha}\right)$ as :

$$
\rho_{P}^{G S}(\boldsymbol{r})=\sum_{\mu, v} S_{\mu \nu} \chi_{\mu}(\boldsymbol{r}) \chi_{\nu}(\boldsymbol{r}) ; \rho_{P}^{E S}(\boldsymbol{r})=\sum_{\mu, v} T_{\mu \nu} \chi_{\mu}(\boldsymbol{r}) \chi_{\nu}(\boldsymbol{r})
$$

with the further condition that atomic indexes $\mu$ and $v$ belong to atoms that are part of the smallest asymmetric fragment ensemble $(\mathbb{A}): \mu, v \in \mathbb{A}$. In these latter equations the suffix " $P$ " stands for "partial", and the $S_{\mu \nu}$ and $T_{\mu \nu}$ are elements of the GS and ES density matrixes written in the basis of atomic orbitals. The resulting electron density variation of a given moiety upon excitation can be written as follows (see ref. 24):

$$
\begin{gathered}
\Delta \rho_{P}(\boldsymbol{r})=\rho_{P}^{E S}-\rho_{P}^{G S} \\
\rho_{P}^{+}(\boldsymbol{r})= \begin{cases}\Delta \rho_{P}(\boldsymbol{r}) & \text { if } \Delta \rho_{P}(\boldsymbol{r})>0 \\
0 & \text { if } \Delta \rho_{P}(\boldsymbol{r}) \leq 0\end{cases} \\
\rho_{P}^{-}(\boldsymbol{r})= \begin{cases}\Delta \rho_{P}(\boldsymbol{r}) & \text { if } \Delta \rho_{P}(\boldsymbol{r})<0 \\
0 & \text { if } \Delta \rho_{P}(\boldsymbol{r}) \geq 0\end{cases} \\
\boldsymbol{R}_{P}^{+}=\frac{\int \rho_{P}^{+}(\boldsymbol{r}) \boldsymbol{r} d \boldsymbol{r}}{\int \rho_{P}^{+}(\boldsymbol{r}) d \boldsymbol{r}} \\
\boldsymbol{R}_{P}^{-}=\frac{\int \rho_{P}^{-}(\boldsymbol{r}) \boldsymbol{r} d \boldsymbol{r}}{\int \rho_{P}^{-}(\boldsymbol{r}) d \boldsymbol{r}} \\
{ }_{\boldsymbol{P}_{\boldsymbol{C}} \boldsymbol{T}}=\boldsymbol{R}^{+}-\boldsymbol{R}^{-} .
\end{gathered}
$$

This descriptor will be referred to as "partial $D_{C T}$ " $\left({ }^{P} D_{C T}\right)$ hereafter. This approach requires a previous knowledge about the nature of the charge-transfer transition since the user has to define the subset of atoms that makes up the smallest asymmetric fragment, thereby adding possible artifacts to the final result including double counting coming from specific molecular fragments.

Here we propose a more general way to compute the particle-hole distance to consider the average of all possible distances between $\rho^{+}(\boldsymbol{r})$ and $\rho^{-}(\boldsymbol{r})$ density distributions, that is:

$$
{ }^{A} D_{C T}=\frac{\iint \mathbb{C}\left(\boldsymbol{r}, \boldsymbol{r}^{\prime}\right)\left|\boldsymbol{r}-\boldsymbol{r}^{\prime}\right| d \boldsymbol{r} d \boldsymbol{r}^{\prime}}{\iint \mathbb{C}\left(\boldsymbol{r}, \boldsymbol{r}^{\prime}\right) d \boldsymbol{r} d \boldsymbol{r}^{\prime}}
$$


Here $\mathbb{C}$ designates a weight, which has been defined based on the following considerations:

i. it has to contain the information related to both $\rho^{+}(\boldsymbol{r})$ and $\rho^{-}(\boldsymbol{r})$;

ii. the distances between maxima of $\rho^{+}(\boldsymbol{r})$ and $\rho^{-}(\boldsymbol{r})$ should be the dominant terms.

iii. the distance between two points of describing the same density distribution (that is the hole or the electron) should have no weight;

In order to satisfy these constraints, the $\mathbb{C}$ coefficient is defined as follows:

$$
\mathbb{C}\left(\boldsymbol{r}, \boldsymbol{r}^{\prime}\right)=\rho_{+}(\boldsymbol{r}) \rho_{-}\left(\boldsymbol{r}^{\prime}\right)
$$

Substituting Equation 21 into Equation 20 we get:

$$
{ }^{A} D_{C T}=\frac{\iint \rho_{+}(\boldsymbol{r}) \rho_{-}\left(\boldsymbol{r}^{\prime}\right)\left|\boldsymbol{r}-\boldsymbol{r}^{\prime}\right| d \boldsymbol{r} d \boldsymbol{r}^{\prime}}{\iint \rho_{+}(\boldsymbol{r}) \rho_{-}\left(\boldsymbol{r}^{\prime}\right) d \boldsymbol{r} d \boldsymbol{r}^{\prime}}
$$

We stress here that while $D_{C T}$ (and ${ }^{P} D_{C T}$ as well) have a vectorial character, being the difference between to positions $\boldsymbol{R}^{+}$and $\boldsymbol{R}^{-}$, the ${ }^{A} D_{C T}$ is a scalar.

One can show that ${ }^{A} D_{C T}$ may be considered as an upper bound of the module of $D_{C T}$. In fact, starting from Eq 1 and substituting Eq 5 and 6 in it, we obtain:

$$
\begin{gathered}
\left|D_{C T}\right|=\left|\boldsymbol{R}^{+}-\boldsymbol{R}^{-}\right|=\left|\frac{\int \rho^{+}(\boldsymbol{r}) \boldsymbol{r} d \boldsymbol{r}}{\int \rho^{+}(\boldsymbol{r}) d \boldsymbol{r}}-\frac{\int \rho^{-}(\boldsymbol{r}) \boldsymbol{r} d \boldsymbol{r}}{\int \rho^{-}(\boldsymbol{r}) d \boldsymbol{r}}\right| \\
=\left|\frac{\int \rho_{-}\left(\boldsymbol{r}^{\prime}\right) d \boldsymbol{r}^{\prime} \int \rho_{+}(\boldsymbol{r}) \boldsymbol{r} d \boldsymbol{r}-\int \rho_{+}(\boldsymbol{r}) d \boldsymbol{r} \int \rho_{-}\left(\boldsymbol{r}^{\prime}\right) \boldsymbol{r}^{\prime} d \boldsymbol{r}^{\prime}}{\int \rho_{-}\left(\boldsymbol{r}^{\prime}\right) d \boldsymbol{r}^{\prime} \int \rho_{+}(\boldsymbol{r}) d \boldsymbol{r}}\right| \\
=\left|\frac{\int \rho_{+}(r) \rho_{-}\left(r^{\prime}\right) r d r d \boldsymbol{r}^{\prime}-\int \rho_{+}(\boldsymbol{r}) \rho_{-}\left(\boldsymbol{r}^{\prime}\right) \boldsymbol{r}^{\prime} d r d \boldsymbol{r}^{\prime}}{\int \rho_{+}(\boldsymbol{r}) \rho_{-}\left(\boldsymbol{r}^{\prime}\right) d \boldsymbol{r}^{\prime} d \boldsymbol{r}}\right|=\left|\frac{\int \rho_{+}(r) \rho_{-}\left(r^{\prime}\right)\left(r-r^{\prime}\right) d r d \boldsymbol{r}^{\prime}}{\int \rho_{+}(\boldsymbol{r}) \rho_{-}\left(\boldsymbol{r}^{\prime}\right) d \boldsymbol{r}^{\prime} d \boldsymbol{r}}\right|
\end{gathered}
$$

Comparing Eq 23 with Eq 22, it is worth to notice that

$$
\left|D_{C T}\right| \leq{ }^{A} D_{C T}
$$

since the ${ }^{A} D_{C T}$ computes an average of all possible distances, unlike the $D_{C T}$ index, which on the contrary estimates the distance between two averaged points.

In practice, the integration is performed as a sum on a large but finite number of grid points on which difference densities are computed. Nonetheless, in the case of ${ }^{A} D_{C T}$, this direct procedure may be impractical due to their large amount and it is therefore possible to compute the ${ }^{A} D_{C T}$ using a discrete number of condensed charges that in our case will be the atomic charges obtained according to some partitioning scheme (like for example Mulliken's or Hirshfeld's procedures), in analogy to equations 8-12. This type of procedure has already been applied to the $D_{C T}$ and ${ }^{P} D_{C T}{ }^{11,24,45}$ and the corresponding equations for the ${ }^{A} D_{C T}$ are: 


$$
\begin{gathered}
\Delta Q_{i}=Q_{i}^{E S}-Q_{i}^{G S} \\
Q_{i}^{+}= \begin{cases}=\Delta Q_{i} & \text { if } \Delta Q_{i}>0 \\
=0 & \text { if } \Delta Q_{i} \leq 0\end{cases} \\
Q_{i}^{-}= \begin{cases}=\Delta Q_{i} & \text { if } \Delta Q_{i}<0 \\
=0 & \text { if } \Delta Q_{i} \geq 0\end{cases} \\
{ }^{A} D_{C T}=\frac{\sum_{i} \sum_{j} Q_{i}^{+} Q_{j}^{-}\left|\boldsymbol{r}_{i}-\boldsymbol{r}_{j}\right|}{\sum_{i} \sum_{j} Q_{i}^{+} Q_{j}^{-}}
\end{gathered}
$$

\section{Computational Details}

Ground state structures and vertical excitation energies were computed, respectively, at the density functional theory (DFT) and at the time-dependent DFT (TD-DFT) levels, ${ }^{47-48}$ using the PBE0 functional ${ }^{49}$ and the $6-31 \mathrm{G}(\mathrm{d})$ basis set. ${ }^{50}$ All optimized structures are given in the Supporting Information. All calculations have been performed using the Gaussian16

software, ${ }^{22}$ while the $D_{C T}$, the ${ }^{P} D_{C T}$ and the ${ }^{A} D_{C T}$ have been evaluated using an in-house developed and freely available code. ${ }^{51}$ Ground and excited state densities were generated using the cubegen and cubeman utilities of the Gaussian16 Package applying default options for grids. The electronic densities considered here are all relaxed densities. ${ }^{52}$

In this paper we considered in particular the properties of those excited $\mathrm{S}_{n}$ states $(n=1, \ldots)$ that have a non-negligible oscillator strength, and that, as a consequence, have an appreciable probability to be populated upon light irradiation.

\section{Results and discussion}

In order to validate and compare ${ }^{A} D_{C T}$ with previously developed descriptors, we firstly considered a set of test case molecules, prototypes of systems containing donor and/or acceptor groups, and spectroscopically well characterized ${ }^{53}$ (1a to $\mathbf{3}$ in Figure 1). For a subset of these model compounds the index was computed both using electron densities distributions and Mulliken atomic charges. Then, we have analyzed a set of symmetric push-pull molecules (4-11 in Figure 1).

We recall here that the aim of the present work is to compare different descriptors and not to investigate the ability of the underlying computational approach (i.e. here of the selected exchange and correlation functional) to capture the photophysics of the systems under analysis. 


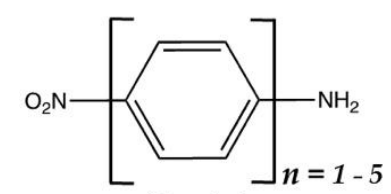

(1a-1e)

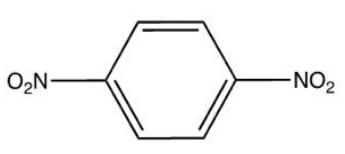

(2)

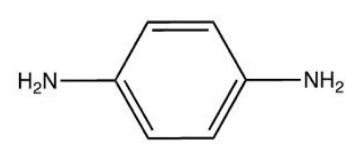

(3)

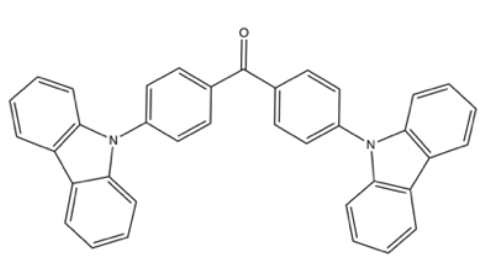

Cz2BP-H (4)

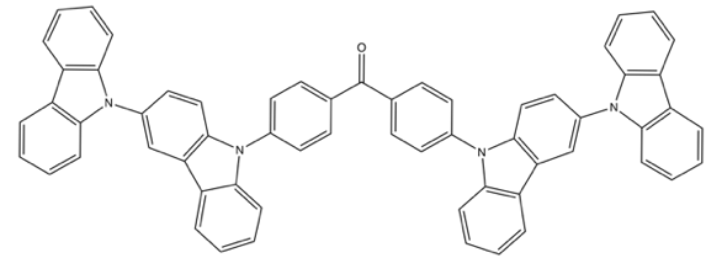

Cz2BP-R (5)

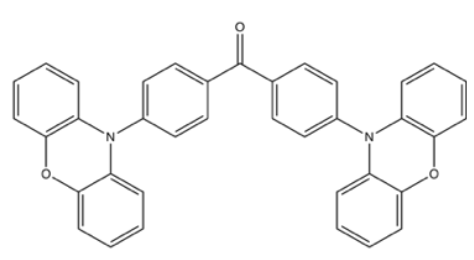

$\operatorname{Px} 2 B P(6)$

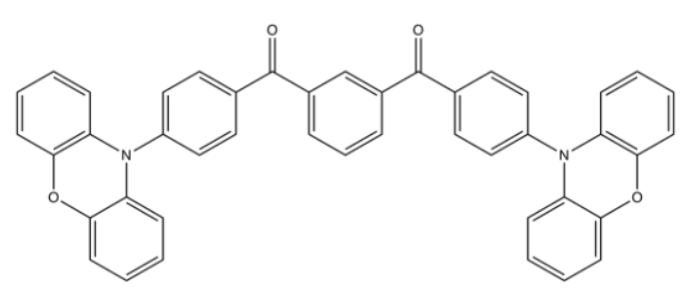

$m-\mathbf{P} 2 B B P(7)$

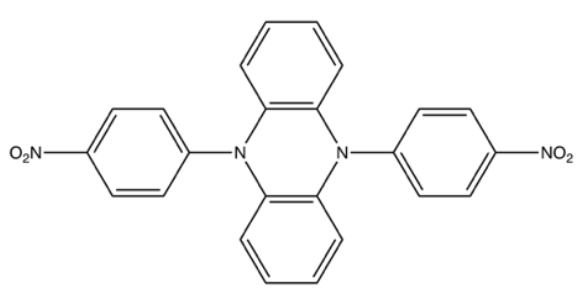

nA ?.Nก, (9)

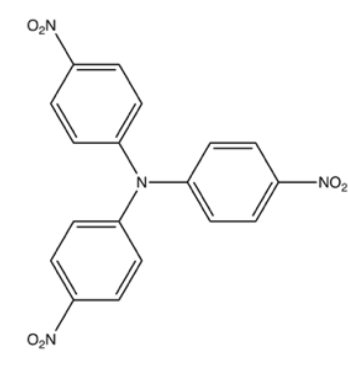

nA 2-NO, (10)

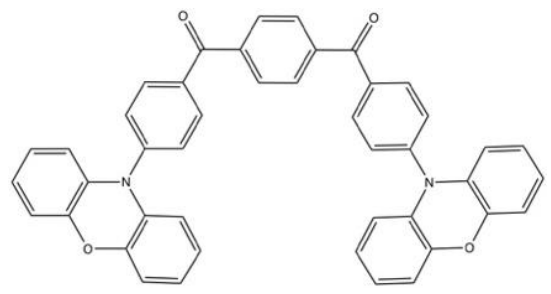

$p$-Px2BBP (8)

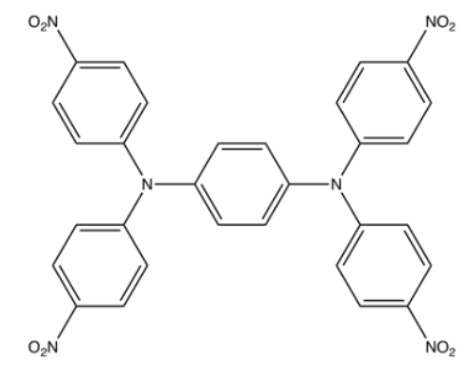

กธ 1 -Nn, (11)

Figure 1. Schematic representation of the model compounds used as donor-acceptor (D-A, 1a-1e for $\mathrm{n}=1,5$ ), acceptor-acceptor (A-A, 2), and donor-donor (D-D, 3 ) together with structural formulae of the Butterfly-shaped benzophenone derivatives (4-8) and of $\mathrm{DA}_{2}-\mathrm{NO}_{2}, \mathrm{DA}_{3}-\mathrm{NO}_{2}$ and $\mathrm{DA}_{4}-\mathrm{NO}_{2}$ molecules (9-11).

We have first considered three of the systems depicted in Figure 1, namely, those possessing a single phenyl ring acting as a bridge, with in para positions either two electron donors (3), or two electron acceptors (2), or one electron donor and one electron acceptor groups (1a).

For these systems we computed the $D_{C T},{ }^{P} D_{C T}$ and ${ }^{A} D_{C T}$ indexes using both densities and Mulliken charges, as reported in Figure S1 and Table S1. As the relationship between CT indexes computed from densities and partial charges is well established, ${ }^{24,45}$ hereafter we present only the analysis carried out with the latter method, as it is easier to implement and perform. This relationship is discussed in more detail in the Section S1 of the Supporting 
Information. Analysis of ${ }^{P} D_{C T}$ has been performed considering the $\mathrm{Ph}-\mathrm{NO}_{2}$ and $\mathrm{Ph}-\mathrm{NH}_{2}$ moieties as acceptor and donor fragments, respectively.

We considered only the lowest, bright (i.e. with a sizable oscillator strength) charge transfer excitations in all cases. These excitations correspond to the first electronic transition $\left(\mathrm{S}_{1} \leftarrow \mathrm{S}_{0}\right)$ for $p$-phenylenediamine (3), and to the second $\left(\mathrm{S}_{2} \leftarrow \mathrm{S}_{0}\right)$ and to the fifth $\left(\mathrm{S}_{5} \leftarrow \mathrm{S}_{0}\right)$ transitions for $p$-nitroaniline (1a) and $p$-dinitrobenzene (2), respectively. All these lowest lying CT states correspond mainly to a one-electron excitation from HOMO to the LUMO as reported in Table 1.

Table 1 Isosurface representation of the HOMO and LUMO (contour value 0.04 a.u., positive and negative values represented in yellow and blue respectively) and $\Delta \boldsymbol{\rho}$ (contour value 0.0004 for a.u., positive and negative values represented in cyan and pink respectively), computed for model systems $\mathbf{1 a}, \mathbf{2}$ and $\mathbf{3}$. The representation with charges is reported in the rightmost column $(\Delta q<0$ in red, $\Delta q>0$ in green).

\begin{tabular}{|c|c|c|c|c|c|c|c|}
\hline & $\begin{array}{l}\text { E / } \\
\text { eV }\end{array}$ & $\begin{array}{l}\text { f / } \\
\text { a.u. }\end{array}$ & НОМО & LUMO & $\Delta \rho$ & $\Delta \boldsymbol{q}$ & $\Delta \boldsymbol{q}$ \\
\hline $1 \mathrm{a}$ & 4.30 & 0.35 & & & & & \\
\hline 2 & 4.43 & 0.02 & & & & & \\
\hline 3 & 4.45 & 0.05 & & & & & \\
\hline
\end{tabular}

All computed values for the adopted descriptors (see Fig. S1 and Table S1 of the Supporting Information) are smaller than the physical distance between the donor and/or acceptor groups, evaluated here as the distance between their nitrogen atoms $(5.76,2.83$, and $2.81 \AA$ for molecules 1a, $\mathbf{2}$ and 3, respectively). This finding is reasonable due to the electron delocalization of both the HOMO and the LUMO on the phenyl bridge (Table 1), which reduces the $\mathrm{CT}$ distance of the transition.

The values computed with ${ }^{A} D_{C T}$ are close for the three molecules because in all these cases the hole (the HOMO in a single-excitation picture) is mainly localized on the $\mathrm{Ph}$ ring. Molecules containing the nitro group (i.e. molecules 1a and 2) display the highest values 
because their LUMOs are mainly localized on their $\mathrm{NO}_{2}$ moieties, whereas (even when it is present) the contribution of amino groups seems negligible.

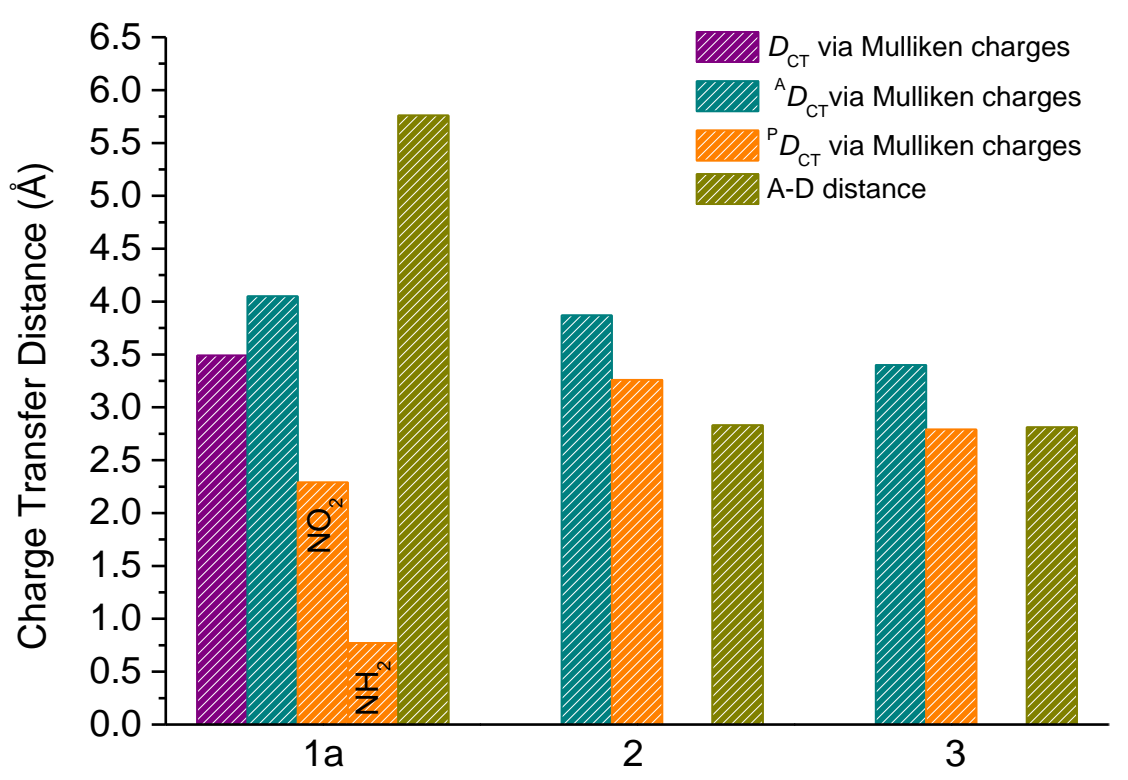

Figure 2. Computed $D_{C T}^{P} D_{C T}$ and ${ }^{A} D_{C T}$ values (in $\AA$ ) for molecules 1a-3.

Regarding molecule 1a, $D_{\mathrm{CT}}$ achieves a value of $3.49 \AA$, lower than the physical distance (5.76 $\AA$, see Figure 2). As expected, we calculated a null $D_{C T}$ for molecule $\mathbf{2}$ and $\mathbf{3}$ because of their symmetric structure. Because of that, an analysis of the ${ }^{P} D_{\mathrm{CT}}$ has been performed to estimate the CT in these two cases. Such values (Figure 2 and Table S1) decrease as expected in going from nitro to amino substituted. Referring ${ }^{P} D_{\mathrm{CT}}$ values, it is possible to observe a qualitatively consistent trend with molecules 1a-3. Thanks to an averaged approach, it is possible to quantify the $\mathrm{CT}$ entity even in the case of an overall zero $D_{\mathrm{CT}}$. The ${ }^{A} D_{\mathrm{CT}}$ yields results higher than $D_{\mathrm{CT}}$ and ${ }^{P} D_{\mathrm{CT}}$, as expected (see Eqn. 24).

To further compare the different descriptors and their ability to describe CT transitions, we considered the push-pull dyad 1a (Figure 1) and enlarged the spacer length by increasing the number of phenyl rings from 1 (1a) to 5 (1e). In all cases the lowest and bright $\mathrm{CT}$ transition corresponds to a $\mathrm{HOMO} \rightarrow \mathrm{LUMO}$ excitation calculated as the first excited state for every system, except for 1a (see before). The computed HOMO and LUMO together with corresponding difference in density and in charges are reported in Table S2. Already from a simple visual inspection of the MOs involved in the CT transition it is clear that, due to the orbital delocalization of the donor moiety (see HOMO orbitals in Table S2), the effective CT distance must be smaller than the physical distance between the donor $\left(-\mathrm{NH}_{2}\right)$ and the 
acceptor $\left(-\mathrm{NO}_{2}\right)$. The latter is very localized independently from the number of the phenyl spacers (Table S2 of the Supporting Information, LUMO orbitals).

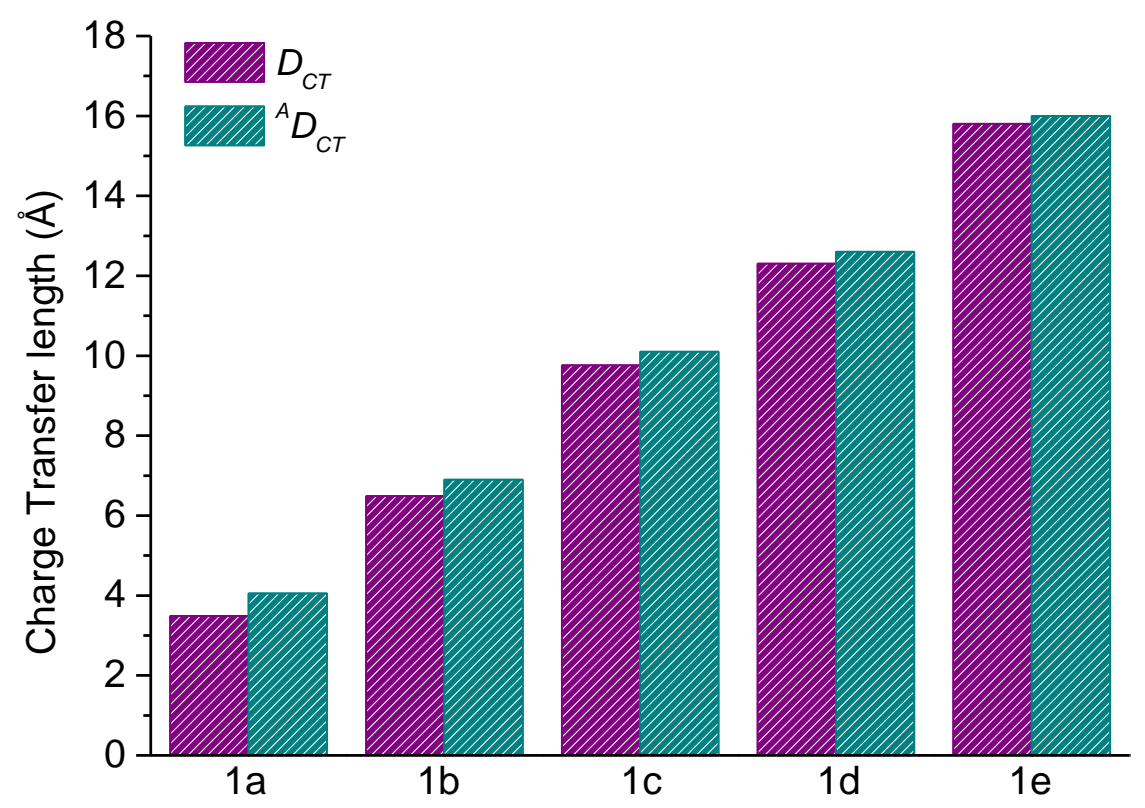

Figure 3. Bar diagram of $D_{C T}$ and ${ }^{A} D_{C T}$ values (in $\AA$ ) calculated from atomic Mulliken charges.

The $D_{C T}$ and ${ }^{A} D_{C T}$ values (Figure 3) consistently quantify this observation: whatever the method used to calculate the CT distance, the values increase adding a phenyl group in the range from $3.50 \AA$ (for molecule 1a) to $16.00 \AA$ (for molecule 1e). Furthermore, both indexes $\left(D_{C T}\right.$ and $\left.{ }^{A} D_{C T}\right)$ provide values that are quantitatively equivalent (Figure 3 ) and their (small) difference decrease upon elongation of the phenyl chain (see Table S3 and Section S2). Upon addition of each phenyl unit the physical distance between A and D increases of ca. $4.33 \AA$, yet the corresponding increment in the CT distance is about $3.00 \AA$ independently on the $D_{C T}$ type considered (as graphically represented by the normalized $D_{C T}$ plot reported in Figure S2, whose values are collected in Table S4). Indeed, upon addition of the phenyl rings both the hole and the electron become more delocalized along the aromatic chain. Reassuringly, both indexes are able to capture this feature both qualitatively and quantitatively.

Next, as real DA symmetric molecules we have selected the push-pull systems molecules 411 represented in Figure 1. Experimentally, these "butterfly-shaped" molecules (4-8 in Figure 1) are interesting since an efficient thermal activated delayed fluorescence (TADF) ${ }^{54-}$ 55 can be achieved, making them promising for application as organic light-emitting diodes 
(OLEDs). Moreover, computational and experimental studies have demonstrated that the careful molecular design of benzophenone derivatives based on Donor-Acceptor-Donor (DA-D) structure is useful to produce organic luminophores with efficient full-color TADF emission. ${ }^{56}$ Previous quantum chemical calculations performed on these compounds suggested that the modulation of the intramolecular charge-transfer (ICT) character of the transitions correlates with a change in the emission wavelength. ${ }^{56}$ Therefore, the quantification of $\mathrm{CT}$ is of the utmost importance for the design of new compounds.

Systems 9-10 (Figure 1) are also promising for OLEDs applications ${ }^{57-58}$ while the $\mathrm{DA}_{4}-\mathrm{NO}_{2}$ (Molecule $\mathbf{1 1}$ in Figure 1) has been chosen since it contains a structural motif often found in hole transport materials applied for a wide range of technologies, from photocopiers to electroluminescent display devices. ${ }^{59}$

The lowest energy $\mathrm{S}_{1} \leftarrow \mathrm{S}_{0}$ excitations correspond essentially to a one-electron $\mathrm{HOMO} \rightarrow$ LUMO transition for all the butterfly molecules investigated here (4-8, Figure 1). These transitions correspond to an intermolecular charge transfer (ICT) as the nature of the HOMO and LUMO distributions shows (see Table 2). Indeed, for all the compounds the LUMO is predominantly located on the central electron-accepting benzophenone core, whereas the HOMO is mainly localized on the electron-donating peripheral carbazole or phenoxazine substituents due to the highly twisted geometry of the molecules which renders coupling of the donor and of acceptor constituents negligible. Indeed, for compounds $\mathbf{4}$ and $\mathbf{5}$ the dihedral angles between the phenyl rings of the benzophenone unit and adjacent carbazoles are calculated to be around $50^{\circ}$ and for 6,7 and 8 with phenoxazines to be around $70^{\circ}$. Consistently, by analysis of oscillator strengths (Table S5) it may be noted that the $\mathrm{S}_{1}$ states for $\mathbf{4}$ and $\mathbf{5}$ have relatively large oscillator strengths ( $\mathrm{f}=0.43$ and 0.25 , respectively), which tend to decrease upon substitution with more sterically-hindered phenoxazine donor units ( $\mathrm{f}=0.04-0.10$ for compounds 6-8).

As expected from the symmetry of these molecules (space group $\mathrm{C}_{2 \mathrm{~V}}$ ), the $D_{C T}$ values do not reflect the evident CT nature of the lowest-energy transitions. Indeed, $D_{C T}$ is null for $\mathbf{5}$ or achieves values which do not exceed $2.00 \AA$ for the other molecules, but $8\left(D_{C T}=3.57 \AA\right)$. 
Table 2. Representation of HOMO and LUMO orbitals involved in the CT transition for butterfly-shaped molecules.

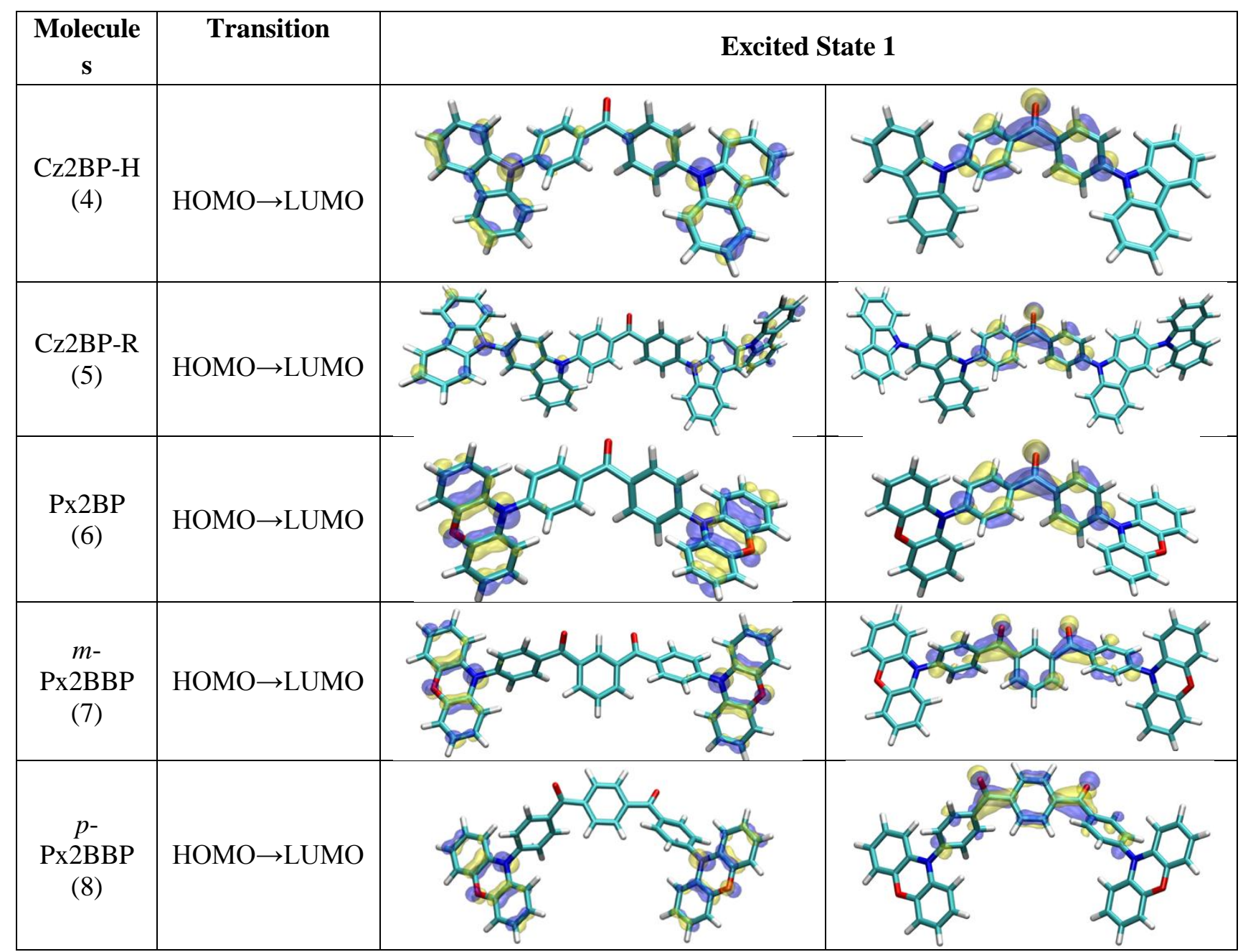

On the other hand, as expected, the ${ }^{P} D_{\mathrm{CT}}$ index is able to quantify the CT entity for all the benzophenone derivatives, as it is straightforward to define the two asymmetric D-A fragments, each of them consisting of one donor moiety and the same acceptor group, which corresponds for all molecules to the benzophenone core (Figure S3). Using this latter definition, we computed the ${ }^{P} D_{C T}$ values for all butterfly molecules, collected in Figure 4 (upper panel) and reported in Table S5. 

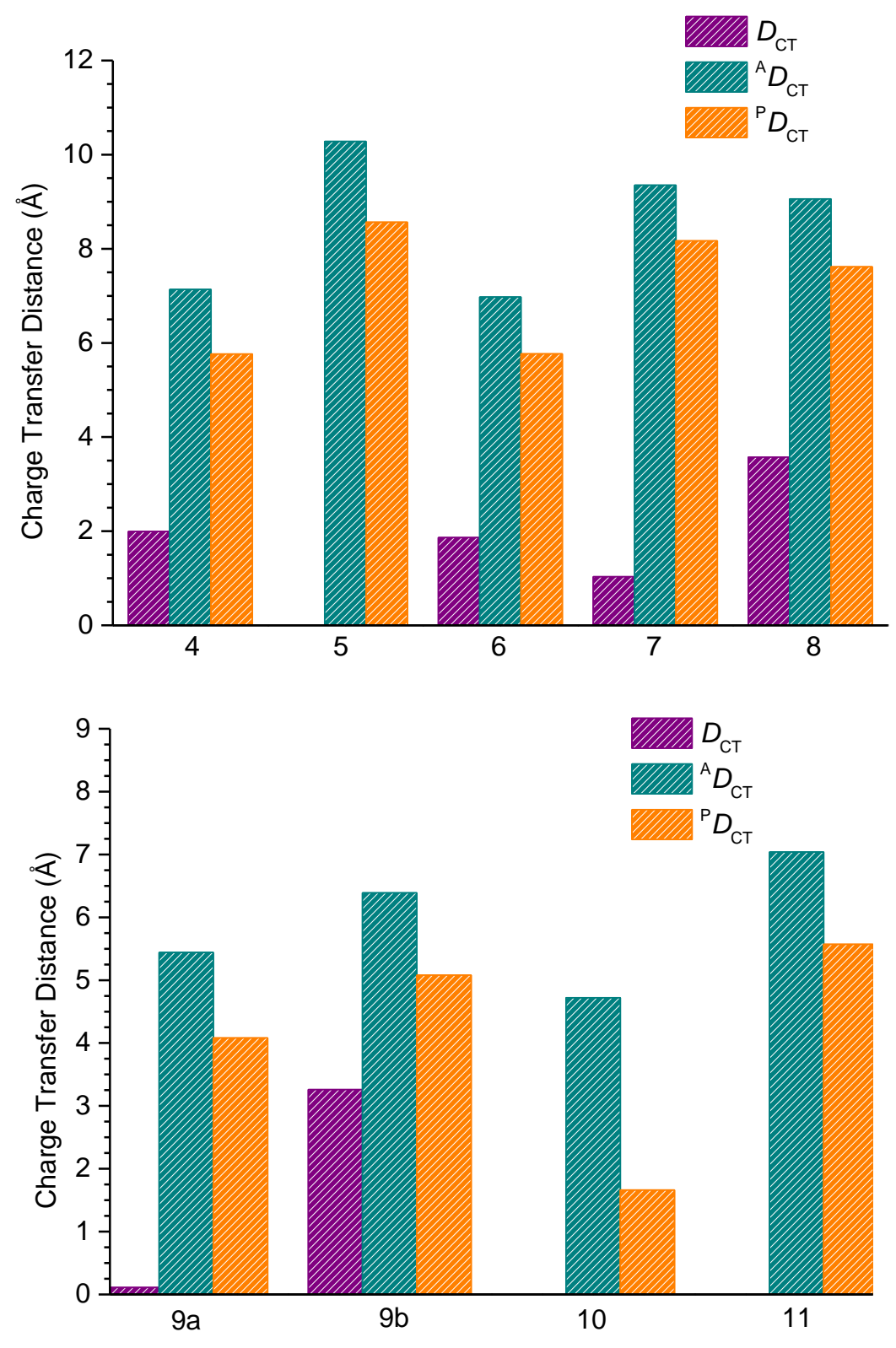

Figure 4. Computed $D_{\mathrm{CT}},{ }^{\mathrm{A}} D_{\mathrm{CT}}$ and ${ }^{\mathrm{P}} D_{\mathrm{CT}}$ values (in $\AA$ ) for molecules 4-8 (upper panel) and for molecules 9a-11 (bottom panel).

Reasonably, having a similar structure, molecules $\mathbf{4}$ and $\mathbf{6}$ present the same ${ }^{P} D_{C T}$ value $(5.76$ $\AA$ ): the carbazole (for 4) or phenoxazine (for 6) lateral groups act as donors towards the acceptor benzophenone core (see Figure S3). Molecules 5 and $\mathbf{7}$ which are characterized by large sterically-hindered peripheral moieties, show the highest ${ }^{P} D_{\mathrm{CT}}$, ranging from $8.00 \AA$ to $8.50 \AA$. As a matter of fact, the strong conjugated residues present in these systems allow an efficient intermolecular charge transfer, notwithstanding a clear physical distance between D and A. The same applies to the molecule 8 , whose ${ }^{P} D_{\mathrm{CT}}$ achieves a value of $7.61 \AA$. Firstly, it is worth to notice that we obtained generally higher ${ }^{A} D_{C T}$ values ( $\geq 7 \AA$, see Figure 4 and Table S5), in accordance with the qualitative analysis of HOMO and LUMO orbitals, 
which are localized on distinct and far apart atoms of the molecule. Moreover, we obtained the same consistent trend individuated by the ${ }^{P} D_{C T}$ analysis. The highest ${ }^{A} D_{C T}$ values are found for molecules 5, 7 and 8 (9.0-10.0 $\AA$ ), while for molecules 4 and $\mathbf{6}$ the ${ }^{A} D_{C T}$ values decrease to about 7.0 $\AA$. By the analysis of the bar diagram in Figure 4 (upper panel), it could be also pinpointed that the ${ }^{A} D_{C T}$ values are higher than ${ }^{P} D_{C T}$ ones for all considered molecules. As mentioned, the explanation stems from the mathematical definition of the averaged descriptor, which takes into account all possible physical distances between opposite charges and does not estimate a unique distance between two averaged barycenters of charges.

Contrary to the symmetric systems 1-8, in 9-11 the peripheral nitro-substituted phenyl rings act as electron-accepting moieties while the electron-donating core is represented by a substituted di-phenyl hydrophenazine moiety, a nitrogen atom and a di-amino phenyl group for $\mathrm{DA}_{2}-\mathrm{NO}_{2}(\mathbf{9}), \mathrm{DA}_{3}-\mathrm{NO}_{2}(\mathbf{1 0})$ and $\mathrm{DA}_{4}-\mathrm{NO}_{2}(\mathbf{1 1})$ respectively (Figure $\left.\mathrm{S} 4\right)$.

All corresponding optimized structures are collected in section S3 of the Supporting Information. It is important to point out here that, at the ground state, two distinct stable conformers exist for the $\mathrm{DA}_{2}-\mathrm{NO}_{2}$ molecule, i.e. a twist (9a) and a boat (9b) conformation. The latter is characterized by a boat shape disposition of di-phenyl hydrophenazine core and it is more stable of $1.40 \mathrm{kcal} / \mathrm{mol}$ than the twist conformer, with the nitro substituted phenyl rings roughly orthogonal to the hydrophenazine core. Notably, the lowest-energy bright symmetric CT transitions are not of the HOMO $\rightarrow$ LUMO type for all these systems and do not always correspond to the $\mathrm{S}_{1} \leftarrow \mathrm{S}_{0}$ excitations. Specifically, we studied the fourth ES (i.e. $\mathrm{S}_{4} \leftarrow \mathrm{S}_{0}$ ) and the seventh ES (i.e. $\mathrm{S}_{7} \leftarrow \mathrm{S}_{0}$ ) for 9a and 10, respectively, and in both cases these transitions correspond to a $\mathrm{HOMO} \rightarrow \mathrm{LUMO}+3$ excitation, as reported in Table 3.

From the molecular orbitals analysis (Table 3), we can notice that the HOMO is mainly localized in the core of the system, while the LUMO for $\mathbf{9 b}$ and $\mathbf{1 1}$ and the LUMO+3 for $\mathbf{9 a}$ and $\mathbf{1 0}$ are predominantly located on the peripheral nitrophenyl groups, acting as acceptors due to the presence of the strong electron withdrawing groups.

Despite the fact that the two conformers of molecule 9 have the same symmetric A-D-A framework, only the twisted one with $\mathrm{D}_{2}$ symmetry has a $D_{C T}$ almost equal to zero. On the contrary, for the boat-like conformer $(9 \mathrm{~b})$ we expected a non-vanishing $D_{C T}$, due to a $\mathrm{C}_{2 \mathrm{~V}}$ symmetric arrangement (3.26 $\AA$ ). For molecules $\mathbf{1 0}$ and $\mathbf{1 1}$ we obtained null $D_{C T}$ values as expected due to their $\mathrm{C}_{3}$ and $\mathrm{D}_{2}$ symmetries, respectively. Compared to these values indeed, the ${ }^{P} D_{C T}$ reveals a more delocalized topology of the excited states. Considering only the 
smallest D-A asymmetric fragment, the partial CT length is somewhat increased achieving values from $1.66 \AA$ (for molecule 10) to $5.50 \AA$ (for molecule 11).

Interestingly, the ${ }^{A} D_{C T}$ values present the same qualitative trend of the ${ }^{P} D_{C T}$ : we find the smallest value for molecule $\mathbf{1 0}$ (4.72 $\AA$ ), while we obtained the highest for molecule 11 (7.03 $\AA$ ), see Table S6 in the Supporting Information. We deem worth pinpointing again that, as in the previous cases and how demonstrated by the mathematical definition, the average values are always higher than the ones calculated by the definition of the barycenters of charges.

Table 3. Representation of HOMO and LUMO orbitals involved in the CT transition for $\mathrm{DA}_{2}-\mathrm{NO}_{2}(\mathbf{9 a}-\mathbf{b}), \mathrm{DA}_{3}-\mathrm{NO}_{2}(\mathbf{1 0})$ and $\mathrm{DA}_{4}-\mathrm{NO}_{2}(\mathbf{1 1})$ molecules. Molecule 9a has $\mathrm{D}_{2}$ symmetry, molecule $\mathbf{9 b}$ has $\mathrm{C}_{2 \mathrm{v}}$ symmetry, molecule $\mathbf{1 0}$ has $\mathrm{C} 3$ symmetry, and molecule $\mathbf{1 1}$ has $\mathrm{D}_{2}$ symmetry.

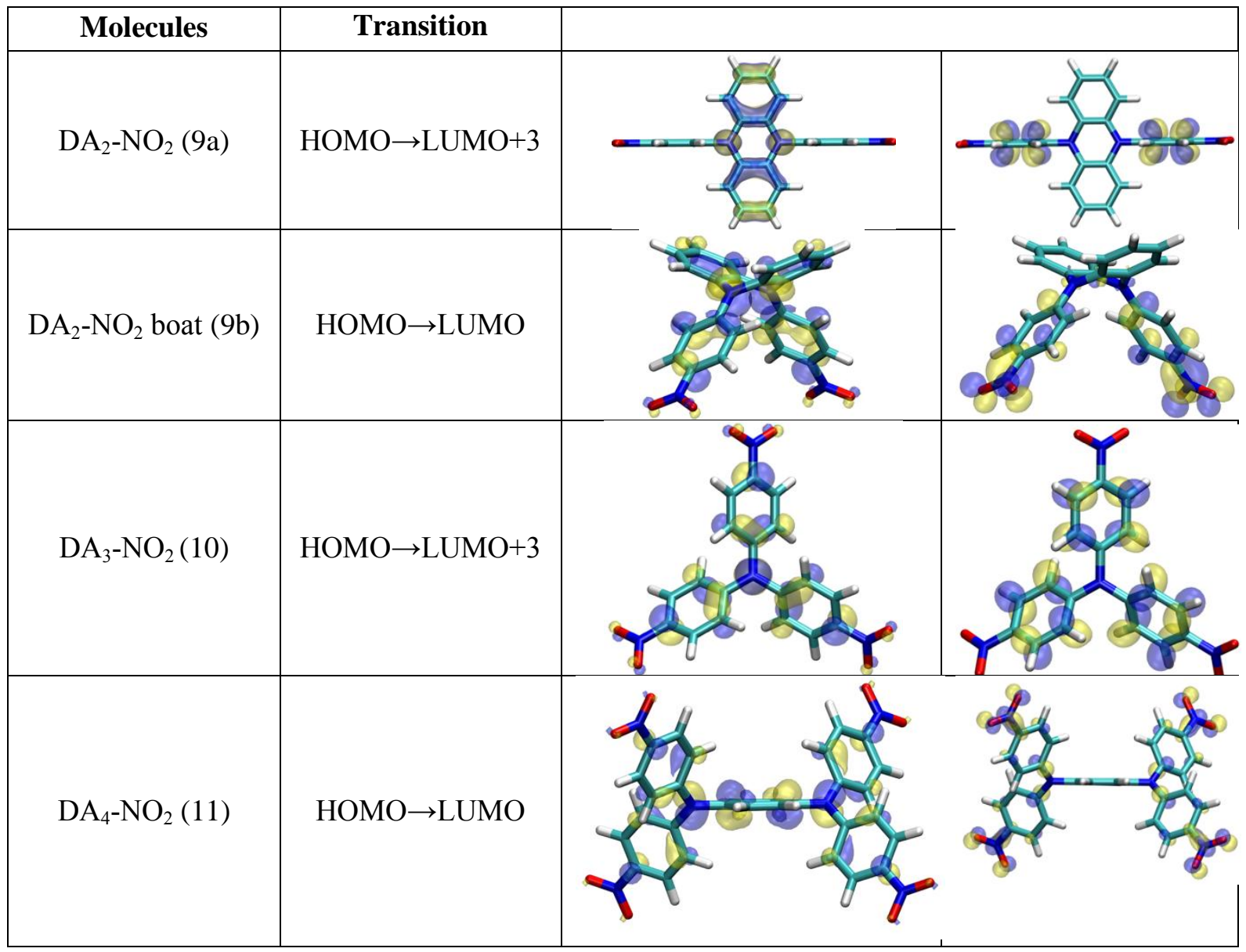

From all these data we can conclude that the ${ }^{A} D_{C T}$ descriptor allows to describe systems rather unfeasible with standard density-based index (e.g. molecules with Donors or Acceptors 
connected by a symmetry operation). Nonetheless for the sake of completeness, we will illustrate here the singular case of a symmetric donor-acceptor (DA) molecule, which well displays the averaged character of the ${ }^{A} D_{C T}$ formulation. This hypothetical model system (Figure 5), hereafter referred to as $\mathrm{AD}-\mathrm{Ph}_{4}-\mathrm{AD}$, shows a peculiar topology possessing an overall $\mathrm{C}_{2 \mathrm{v}}$ symmetry with two symmetry related $\mathrm{NH}_{2}$ residues acting as donors, and two symmetry related acceptor groups $\left(\mathrm{NO}_{2}\right)$. Overall the systems present therefore two symmetry equivalent $\mathrm{D}-\mathrm{A}$ pair. In this case, two $\mathrm{NO}_{2} \leftarrow \mathrm{NH}_{2}$ charge transfers can be considered: one at a short distance, represented by the DA pair of the same phenyl $(\sim 5 \AA)$, and another at longer distance represented by the pair of $\mathrm{D}$ and A placed on the opposite sides of the molecule $(\sim 16 \AA)$, as schematically depicted in Figure 5 .

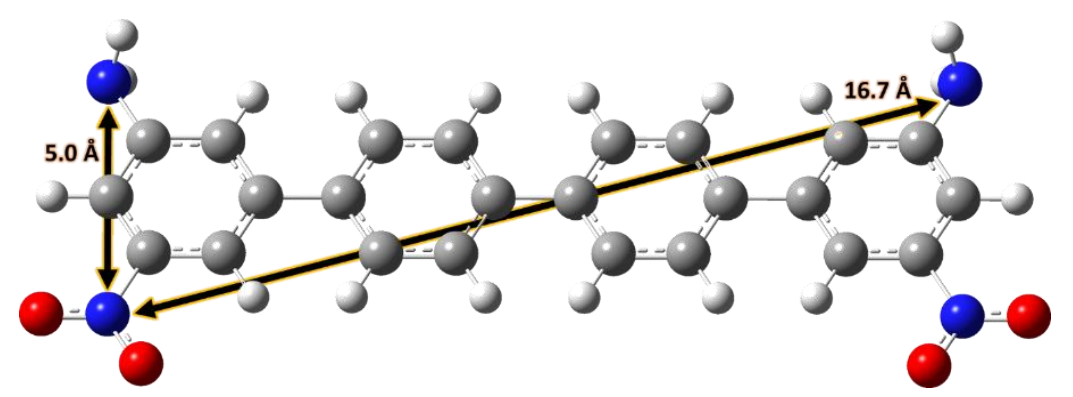

Figure 5. Representation of the $\mathrm{AD}-\mathrm{Ph}_{4}-\mathrm{AD}$ systems $\left(\mathrm{C}_{2 \mathrm{v}}\right.$ symmetry) with two possible $\mathrm{CT}$ distances here measured as the distance between the $\mathrm{N}$ atoms of $-\mathrm{NH}_{2}$ groups and $-\mathrm{NO}_{2}$ groups

In this $\mathrm{C}_{2 \mathrm{v}}$ conformation, the two possible $\mathrm{A} \leftarrow \mathrm{D}$ (i.e. $\mathrm{NO}_{2} \leftarrow \mathrm{NH}_{2}$ ) charge transfers are energetically degenerate. For symmetry reasons, the long-range CT contributions cancel out in a $D_{C T}$ formalism leading to a very small global value independently of the fact that charges or density are used to evaluate it (Table 7). On the contrary, the ${ }^{A} D_{C T}$ formalism yields a value $(\sim 10 \AA)$ which is close to the average of the two possible $\mathrm{NH}_{2} \rightarrow \mathrm{NO}_{2}$ distances $(\sim 5.0$ $\AA$ and $\sim 16.7 \AA$, respectively). 
Table 7. Computed $D_{C T}$ and ${ }^{A} D_{C T}(\AA)$ for the $\mathrm{AD}-\mathrm{Ph}_{4}$ - $\mathrm{AD}$ molecule along with the isosurfaces of the orbitals involved in the transition and difference in ground and excited state densities $\left(\Delta \rho=\rho_{\mathrm{ES}}-\rho_{\mathrm{EG})}\right.$

\begin{tabular}{|c|c|c|c|c|c|c|}
\hline \multicolumn{2}{|c|}{} & $\begin{array}{c}\text { Indexes computed via } \\
\text { electronic Density }\end{array}$ & \multicolumn{2}{c|}{$\begin{array}{c}\text { Indexes computed via } \\
\text { Mulliken Charges }\end{array}$} \\
\hline $\mathbf{S}_{n}$ & $\mathbf{E} / \mathbf{e V}$ & Symmetry & $D_{C T}$ & ${ }^{A} \boldsymbol{D}_{C T}$ & $D_{C T}$ & ${ }^{A} D_{C T}$ \\
\hline & 4.29 & $\mathrm{C}_{2 \mathrm{v}}$ & 1.51 & 9.70 & 3.52 & 9.86 \\
\hline
\end{tabular}

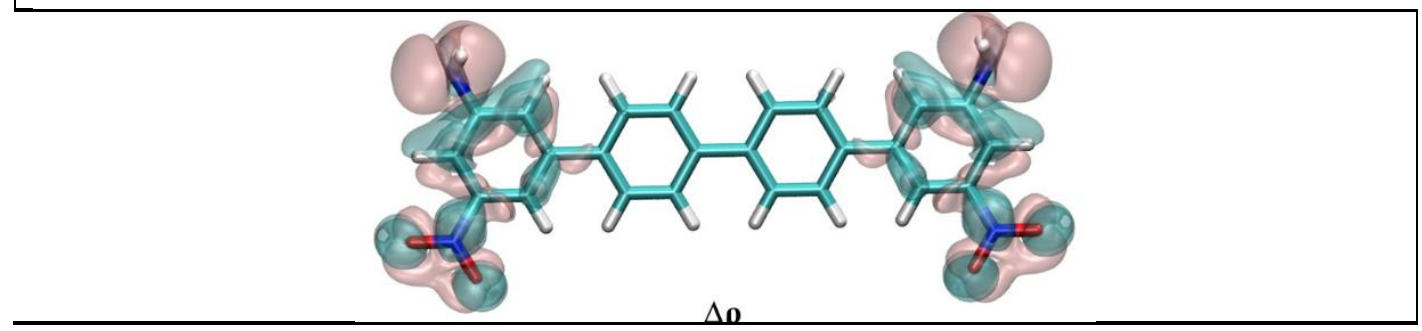

This example clearly shows that the ${ }^{A} D_{C T}$ corresponds to the global average distance of two energetically degenerate CTs and it cannot give insights on the CT distances associated to the different phenomena. If the user aims quantifying separately the two A-D distances, in this case we suggest using the ${ }^{P} D_{\mathrm{CT}}$ method, since in that way one can select the corresponding fragments contributing to the desired transition.

\section{Conclusions}

Here we have presented and discussed a density-based index (called ${ }^{A} D_{C T}$ ) enabling to quantify the charge transfer length associated to an electronic transition. With respect to previously developed density-based indexes like $D_{C T}$ and ${ }^{P} D_{C T}$, the ${ }^{A} D_{C T}$ allows to achieve a description that is independent of the system topology. Indeed, from a theoretical standpoint, this index yields reasonable amounts and distance of charge-transfer even for most symmetric molecules, which are the systems usually not correctly described by density-based descriptors. This descriptor retains the positive aspects of the previous density based descriptors: it has a particularly low computational burden if used in conjunction with atomic charges (for instance Mulliken's ones). Also, it can be applied independently on the level of 
theory adopted (as long as it allows to investigate electronic excited states), thus can be applied "out-of-the-box" both with calculations based on density functional theory as well as on wave-function theory.

For sake of completeness, we also have to pinpoint that the ${ }^{A} D_{C T}$, as the name implies, yields an average value, so it is inherently a scalar (and not a vectorial) quantity. Indeed, contrary to the original $D_{C T}$ it does not provide a physically correlated origin of the displacement of the electron and the hole. As long as donor and acceptor groups are easily identified this does not

represent a significant drawback. Since the ${ }^{A} D_{C T}$ is an average, when applied on systems with two (or more) symmetry equivalent donor-acceptor pairs, it will not provide a value of the intra and inter-pair charge transfer distances individually, but instead their average and thus it requires care in its use and interpretation.

\section{ASSOCIATED CONTENT}

\section{Supporting Information}

Table S1: Computed data for molecules 1a-3.

Table S2: Representation of HOMO and LUMO, density difference and charge difference for molecules 1a-1e.

Table S3: Computed data for molecules 1a-1e.

Table S4: Normalized values of $D_{\mathrm{CT}}$ and ${ }^{\mathrm{A}} D_{\mathrm{CT}}$ for molecules 1a-1e.

Table S5: Computed data for molecules 4-8.

Table S6: Computed data for molecules 9-11.

Section S1: Relationship between indexes computed with partial charges and densities.

Section S2: Observation regarding the oscillator strengths for molecules 1a-1e.

Section S3: Optimized Cartesian coordinates of all investigated molecules.

\section{AUTHOR INFORMATION}

\section{Corresponding author}

*E-mail: $\underline{\text { ilaria.ciofini@chimieparistech.psl.eu }}$

*E-mail: f.muniz-miranda@chimieparistech.psl.eu 


\section{ACKNOWLEDGEMENT}

This project has received funding from the European Research Council (ERC) under the

European Union's Horizon 2020 research and innovation programme (grant agreement No 648558, STRIGES CoG grant).

\section{REFERENCES}

(1) Kraner, S.; Scholz, R.; Koerner, C.; Leo, K. Design Proposals for Organic Materials Exhibiting a Low Exciton Binding Energy. J. Phys. Chem. C. 2015, 119, 22820-22825.

(2) Chen, J.; Yan, M.; Tang, Y.; Yu, J.; Xu, W.; Fu, Y.; Cao, H.; He, Q.; Cheng, J. Rational Construction of Highly Tunable Organic Charge-Transfer Complexes for Chemiresistive Sensor Applications. ACS Appl. Bio Mater. 2019, 2, 3678-3685.

(3) Salzillo,T.; Capos, A.; Mas-Torrent, M. Solution-processed thin films of a charge transfer complex for ambipolar field-effect transistors. J. Mater. Chem. C 2019, 7, 10257-10263.

(4) Cannizzo, A. Ultrafast UV spectroscopy: From a local to a global view of dynamical processes in macromolecules. Phys. Chem. Chem. Phys. 2012, 14, 11205-11223.

(5) Stolow, A.; Bragg, A. E.; Neumark, D. M. Femtosecond time-resolved photoelectron spectroscopy. Chem. Rev. 2004, 104, 1719-1758.

(6) Adamo, C.; Jacquemin, D. The calculations of excited-state properties with Time-Dependent Density Functional Theory. Chem. Soc. Rev. 2013,42, 845-856.

(7) Elliott, P.; Furche, F.; Burke, K. Excited States from Time- Dependent Density Functional Theory. In Reviews in Computational Chemistry. Lipkowitz, K.B.; Cundari, T.R. Eds.; Wiley: Hoboken, NJ, 2008; Vol. 26; p. 91.

(8) Head-Gordon, M.; Grana, A. M.; Maurice, D.; White, C. A. Analysis of electronic transitions as the difference of electron attachment and detachment densities. J. Phys. Chem. 1995, 99, 14261-14270.

(9) Martin, R. L. Natural transition orbitals. J. Chem. Phys. 2003, 118, 4775-4777.

(10) Plasser, F.; Wormit, M.; Dreuw, A. New tools for the systematic analysis and visualization of electronic excitations. I. Formalism. J. Chem. Phys. 2014, 141, 024106.

(11) Le Bahers, T.; Adamo, C.; Ciofini, I. A qualitative index of spatial extent in charge-transfer excitations. J. Chem. Theory Comput. 2011, 7, 2498-2506.

(12) Chibani, S.; Le Guennic, B.; Charaf-Eddin, A.; Laurenta, A.D.; Jacquemin, D. Revisiting the optical signatures of BODIPY with ab initio tools. Chem. Sci. 2013, 4, 1950-1963.

(13) Coe, B.J.; Avramopoulos, A.; Papadopoulos, M.G.; Pierloot, K.; Vancoillie, S.; Reis. H. Theoretical Modelling of Photoswitching of Hyperpolarisabilities in Ruthenium Complexes. Chem. Eur. J. 2013, 19, 15955-15963.

(14) De Sousa, S.; Ducasse, L.; Kauffmann, B.; Toupance, T.; Olivier, C. Functionalization of a Ruthenium-Diacetylide Organometallic Complex as a Next-Generation Push-Pull Chromophore. Chem. Eur. J. 2014, 20, 7017-7024.

(15) De Sousa, S.; Lyu, S.; Ducasse, L.; Toupance, T.; Olivier, C. Tuning visible-light absorption properties of Ru-diacetylide complexes: simple access to colorful efficient dyes for DSSCs. $J$. Mater. Chem. A 2015, 3, 18256-18264.

(16) Olaru, M.; Rychagova, E.; Ketkov, S.; Shynkarenko, Y.; Yakunin, S.; Kovalenko, M.V.; Yablonskiy, A.; Andreev, B.; Kleemiss, F.; Beckmann, J.; Vogt. M. A Small Cationic OrganoCopper Cluster as Thermally Robust Highly Photo- and Electroluminescent Material. J. Am. Chem. Soc. 2020, 142, 373-381.

(17) Laurent, A.D.; Jacquemin, D. Analyzing excited-state processes and optical signatures of a ratiomeric fluorine anion sensor: a quantum look. Sci. China Chem. 2014, 5, 1363-1368. 
(18) Lu, X.; Shao, Y.; Wei, S.; Zhao, Z.; Li, K.; Guo, C.; Wang, W.; Zhang, M.; Guo. W. Effect of the functionalized $\pi$-bridge on porphyrin sensitizers for dye-sensitized solar cells: an in-depth analysis of electronic structure, spectrum, excitation, and intramolecular electron transfer. $J$. Mater. Chem. C 2015, 3, 10129-10139.

(19) Venkatraman, V.; Foscato, M.; Jensen, V.R.; Alsberg, B.K. Evolutionary de novo design of phenothiazine derivatives for dye-sensitized solar cells. J. Mater. Chem. A 2015, 3, 9851-9860.

(20) Olivier, Y.; Sancho-Garcia, J.-C.; Muccioli, L.; D’Avino, G.; Beljonne, D. Computational Design of Thermally Activated Delayed Fluorescence Materials: The Challenges Ahead. $J$. Phys. Chem. Lett. 2018, 9, 6149-6163.

(21) Al Mamun, A.; Toda, M.J.; Lodowski, P.; Jaworska, M.; Kozlowski, P.M. Mechanism of Light Induced Radical Pair Formation in Coenzyme B12-Dependent Ethanolamine AmmoniaLyase. ACS Catal. 2018, 8, 7164-7178.

(22) Frisch, M. J.; Trucks, G. W.; Schlegel, H. B.; Scuseria, G. E.; Robb, M. A.; Cheeseman, J. R.; Scalmani, G.; Barone, V.; Petersson, G. A.; Nakatsuji, H.; Li, X.; Caricato, M.; Marenich, A. V.; Bloino, J.; Janesko, B. G.; Gomperts, R.; Mennucci, B.; Hratchian, H. P.; Ortiz, J. V.; Izmaylov, A. F.; Sonnenberg, J. L.; Williams-Young, D.; Ding, F.; Lipparini, F.; Egidi, F.; Goings, J.; Peng, B.; Petrone, A.; Henderson, T.; Ranasinghe, D.; Zakrzewski, V. G.; Gao, J.; Rega, N.; Zheng, G.; Liang, W.; Hada, M.; Ehara, M.; Toyota, K.; Fukuda, R.; Hasegawa, J.; Ishida, M.; Nakajima, T.; Honda, Y.; Kitao, O.; Nakai, H.; Vreven, T.; Throssell, K.; Montgomery, J. A., Jr.; Peralta, J. E.; Ogliaro, F.; Bearpark, M. J.; Heyd, J. J.; Brothers, E. N.; Kudin, K. N.; Staroverov, V. N.; Keith, T. A.; Kobayashi, R.; Normand, J.; Raghavachari, K.; Rendell, A. P.; Burant, J. C.; Iyengar, S. S.; Tomasi, J.; Cossi, M.; Millam, J. M.; Klene, M.; Adamo, C.; Cammi, R.; Ochterski, J. W.; Martin, R. L.; Morokuma, K.; Farkas, O.; Foresman, J. B.; Fox, D. J. Gaussian 16, Revision C.01, Gaussian, Inc., Wallingford CT, 2016.

(23) Lu, T.; Chen, F. Multiwfn: A multifunctional wavefunction analyzer. J. Comput. Chem. 2012, 33, 580-592.

(24) Campetella, M.; Perfetto, A.; Ciofini, I. Quantifying partial hole-particle distance at the excited state: A revised version of the DCT index. Chem. Phys. Lett. 2019, 714, 81-86.

(25) Sanz García, J.; Maschietto, F.; Campetella, M.; Ciofini, I. Using Density Based Indexes and Wave Function Methods for the Description of Excited States: Excited State Proton Transfer Reactions as a Test Case. J. Phys. Chem. A. 2018, 122, 375-382.

(26) Etienne, T.; Assfeld, X.; Monari, A. Toward a quantitative assessment of electronic transitions" charge-transfer character. J. Chem. Theory Comput. 2014, 10, 3896-3905.

(27) Etienne, T.; Assfeld, X.; Monari, A. New insight into the topology of excited states through detachment/attachment density matrices-based centroids of charge. J. Chem. Theory and Comput. 2014, 10, 3906-3914.

(28) Etienne, T. Transition matrices and orbitals from reduced density matrix theory. J. Chem. Phys. 2015, 142, 244103-17.

(29) Guido, C. A.; Cortona, P.; Adamo, C. Effective electron displacements: A tool for timedependent density functional theory computational spectroscopy. J. Chem. Phys. 2014, 140, 104101-9.

(30) Peach, M.J.G.; Benfield, P.; Helgaker,T.; Tozer, D.J. Excitation energies in density functional theory: An evaluation and a diagnostic test. J. Chem. Phys. 2008, 128, 044118.

(31) Tretiak, S.; Igumenshchev, K., Chernyak, V. Exciton sizes of conducting polymers predicted by time-dependent density functional theory. Phys. Rev. B-Condensed Matter and Materials Physics. 2005, 71, 033201.

(32) Cai, Z. L., Sendt, K., Reimers, J. R. Failure of density-functional theory and time-dependent density-functional theory for large extended $\pi$ systems. J. Chem. Phys. 2002, 117, 5543-5549.

(33) Kuritz, N.; Stein, T.; Baer, R.; Kronik, L. Charge-transfer-like $\pi \rightarrow \pi^{*}$ excitations in timedependent density functional theory: A conundrum and its solution. J. Chem. Theory Comput. 2011, 7, 2408-2415.

(34) Plasser, F.; Thomitzni, B.; Bäppler, S. A.; Wenzel, J., Rehn, D. R.; Wormit, M.; Dreuw, A. Statistical analysis of electronic excitation processes: Spatial location, compactness, charge transfer, and electron-hole correlation. J. Comput. Chem. 2015, 36, 1609-1620. 
(35) Plasser, F.; González, L. Communication: Unambiguous comparison of many-electron wavefunctions through their overlaps. J. Chem. Phys. 2016, 145, 021103.

(36) Mewes, S. A.; Plasser, F.; Krylov, A.; Dreuw, A. Benchmarking Excited-State Calculations Using Exciton Properties. J. Chem. Theory Comput. 2018, 14, 710-725.

(37) Plasser, F.; Lischka, H. Analysis of excitonic and charge transfer interactions from quantum chemical calculations. J. Chem. Theory Comput. 2012, 8, 2777-2789.

(38) Bäppler, S. A.; Plasser, F.;Wormit, M.; Dreuw, A. Exciton analysis of many-body wave functions: Bridging the gap between the quasiparticle and molecular orbital pictures. Physical Review A - Atomic, Molecular, and Optical Physics. 2014, 90, 052521.

(39) Sharifzadeh, S.; Darancet, P.; Kronik, L.; Neaton, J.B. J. Phys. Chem. Lett. 2013, 4, 2197-2201.

(40) Ciofini, I.; Le Bahers, T.; Adamo, C.; Odobel, F.; Jacquemin, D. Through-space charge transfer in rod-like molecules: Lessons from theory. J. Phys. Chem. C. 2012, 116, 1194611955.

(41) Muniz-Miranda, F.; Pedone, A.; Muniz-Miranda, M. Spectroscopic and DFT investigation on the photo-chemical properties of a push-pull chromophore: 4-Dimethylamino-4'-nitrostilbene, Spectrochim. Acta A, 2018, 190, 33-39.

(42) Muniz-Miranda, F.; Minei, P.; Contiero, L.; Labat, F.; Ciofini, I.; Adamo, C.; Bellina, F.; Pucci, A. Aggregation Effects on Pigment Coatings: Pigment Red 179 as a Case Study. ACS Omega 2019, 4, 20315-20323

(43) Improta, R.; Santoro, F.; Blancafort, L. Quantum Mechanical Studies on the Photophysics and the Photochemistry of Nucleic Acids and Nucleobases. Chemical Reviews, 2016, 116, 35403593.

(44) Savarese, M.; Netti, P. A.; Adamo, C.; Rega, N.; Ciofini, I. Exploring the Metric of Excited State Proton Transfer Reactions. J. Phys. Chem. B 2013, 117, 16165-16173.

(45) Jacquemin, D.; Le Bahers, T.; Adamo, C.; Ciofini, I. What is the "best" atomic charge model to describe through-space charge-transfer excitations? Phys. Chem. Chem. Phys. 2012, 14, 5383-5388.

(46) García, G.; Adamo, C.; Ciofini, I. Evaluating push-pull dye efficiency using TD-DFT and charge transfer indices. Phys. Chem. Chem. Phys., 2013,15, 20210-20219.

(47) Heinze, H. H.; Görling, A.; Rösch, N. Efficient method for calculating molecular excitation energies by time-dependent density-functional theory. J. Chem. Phys. 2000, 113, 2088.

(48) Furche, F.; Ahlrichs, R. Adiabatic time-dependent density functional methods for excited state properties. J. Chem. Phys. 2002, 117, 7433.

(49) Adamo, C.; Barone, V. Toward reliable density functional methods without adjustable parameters: The PBE0 model. J. Chem. Phys. 1999, 110, 6158.

(50) Krishnan, R.; Binkley, J. S.; Seeger, R.; Pople, J. Self-consistent molecular orbital methods. A basis set for correlated wave functions. J. Chem. Phys. 1980, 72, 650.

(51) CTM Group, http://www.quanthic.fr.

(52) Maschietto, F.; Campetella, M.; Frisch, M. J.; Scalmani, G.; Adamo, C.; Ciofini, I. How Are the Charge Transfer Descriptors Affected by the Quality of the Underpinning Electronic Density? J. Comput. Chem. 2018, 39, 735-742.

(53) Berry, R. W. H.; Brocklehurst, P.; Burawoy, A. The effect of terminal nitro and amino groups on the electronic spectra of conjugated hydrocarbon systems. Tetrahedron. 1960, 10, 109-117.

(54) Chen, X. K.; Kim, D.; Brédas, J. L. Thermally Activated Delayed Fluorescence (TADF) Path toward Efficient Electroluminescence in Purely Organic Materials: Molecular Level Insight. Acc. Chem. Res. 2018, 51, 2215-2224.

(55) Yang, Z.; Mao, Z.; Xie, Z.; Zhang, Y.; Liu, S.; Zhao, J.; Xu, J.; Chi, Z.; Aldred, M. P. Recent advances in organic thermally activated delayed fluorescence materials. Chem. Soc. Rev. 2017, 46, 915-1016.

(56) Lee, S. Y.; Yasuda, T.; Yang, Y. S.; Zhang, Q.; Adachi, C. Luminous Butterflies: Efficient Exciton Harvesting by Benzophenone Derivatives for Full-Color Delayed Fluorescence OLEDs. Angew. Chem. Int. Ed. 2014, 53, $6402-6406$.

(57) Wong, M. Y.; Zysman-Colman, E. Purely Organic Thermally Activated Delayed Fluorescence Materials for Organic Light-Emitting Diodes. Adv. Mater. 2017, 29, 1605444. 
(58) Lee, J.; Shizu, K.; Tanaka, H.; Nakanotani, H.; Yasuda, T.; Kaji, H.; Adachi, C. Controlled emission colors and singlet-triplet energy gaps of dihydrophenazine-based thermally activated delayed fluorescence emitters. J. Mater. Chem. C. 2015, 3, 2175-2181.

(59) Zhang, J.; Chen, Z.; Yang, L.; Pan, F. F.; Yu, G. A.; Yin, J.; Liu, S. H. Elaborately Tuning Intramolecular Electron Transfer Through Varying Oligoacene Linkers in the Bis(diarylamino) Systems. Sci. Rep. 2016, 6, 36310. 
TOC

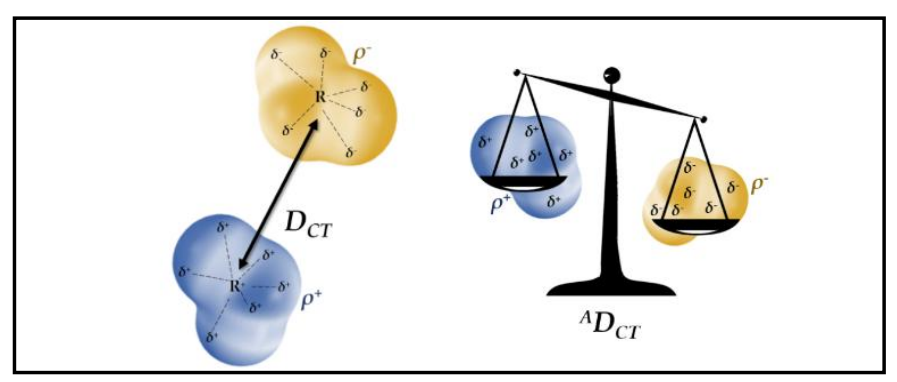

